Bull. Korean Math. Soc. 49 (2012), No. 5, pp. 1067-1079

http://dx.doi.org/10.4134/BKMS.2012.49.5.1067

\title{
UNIFORM AND COUNIFORM DIMENSION OF GENERALIZED INVERSE POLYNOMIAL MODULES
}

\author{
RENYU ZHAO
}

\begin{abstract}
Let $M$ be a right $R$-module, $(S, \leq)$ a strictly totally ordered monoid which is also artinian and $\omega: S \longrightarrow \operatorname{Aut}(R)$ a monoid homomorphism, and let $\left[M^{S, \leq}\right]_{\left[\left[R^{S,} \leq, \omega\right]\right]}$ denote the generalized inverse polynomial module over the skew generalized power series ring $\left[\left[R^{S, \leq}, \omega\right]\right]$. In this paper, we prove that $\left[M^{S, \leq}\right]_{\left[\left[R^{S,} \leq, \omega\right]\right]}$ has the same uniform dimension as its coefficient module $M_{R}$, and that if, in addition, $R$ is a right perfect ring and $S$ is a chain monoid, then $\left[M^{S, \leq}\right]_{\left[\left[R^{S,},, \omega\right]\right]}$ has the same couniform dimension as its coefficient module $M_{R}$.
\end{abstract}

\section{Introduction}

Throughout this paper, $R$ denotes a ring with identity and modules are unitary right $R$-modules. The uniform dimension (resp. couniform dimension) of a module $M_{R}$ will be denoted by $\operatorname{u} \cdot \operatorname{dim}\left(M_{R}\right)\left(\operatorname{resp}\right.$. corank $\left.\left(M_{R}\right)\right)$. We will denote by $\operatorname{End}(R)$ the monoid of ring endomorphisms of $R$, and by Aut $(R)$ the group of ring automorphisms of $R$.

The behavior of the uniform dimension and the couniform dimension of a ring (resp. a module) under various polynomial extensions have been studied by many researchers, such as Shock [20], Varadarajan [19, 22, 23], Grzeszczuk [4], Matczuk [12] and Annin [1,2]. In particular, Annin in [1, 2] obtained results how the uniform dimension and the couniform dimension of a module behaves on inverse polynomial modules. It was proved that for any right $R$ module $M$, u.dim $\left(M\left[x^{-1}\right]_{R[x ; \sigma]}\right)=\mathrm{u} \cdot \operatorname{dim}\left(M_{R}\right)$, and that if, in addition, $R$ is a right perfect ring, then $\operatorname{corank}\left(M\left[x^{-1}\right]_{R[x ; \sigma]}\right)=\operatorname{corank}\left(M_{R}\right)$, where $\sigma \in$ $\operatorname{Aut}(R)$. In [10], as a generalization of inverse polynomial modules, Liu and Cheng introduced the notion of generalized inverse polynomial modules. Many properties of generalized inverse polynomial modules have been explored in recent years, see for example $[6,7,8,10,11]$ and [24]. Motivated by these facts, in this paper, we will generalize Annin's work to generalized inverse

Received June 12, 2011.

2010 Mathematics Subject Classification. Primary 16W60.

Key words and phrases. skew generalized power series ring, generalized inverse polynomial module, uniform dimension, couniform dimension. 
polynomial modules over skew generalized power series rings. We will show that, if $(S, \leq)$ is a strictly totally ordered monoid which is also artinian and $\omega: S \longrightarrow \operatorname{Aut}(R)$ a monoid homomorphism, then for any right $R$-module $M, \mathrm{u} \cdot \operatorname{dim}\left(\left[M^{S, \leq}\right]_{\left[\left[R^{S, \leq, \omega]}\right]\right.}\right)=\mathrm{u} \cdot \operatorname{dim}\left(M_{R}\right)$, and that if, in addition, $R$ is a right perfect ring and $S$ is a chain monoid, then corank $\left(\left[M^{S, \leq}\right]_{\left[\left[R^{S, \leq, \omega]}\right]\right.}\right)=$ corank $\left(M_{R}\right)$.

Let $(S, \leq)$ be a partially ordered set. Recall that $(S, \leq)$ is artinian if every strictly decreasing sequence of elements of $S$ is finite, and that $(S, \leq)$ is narrow if every subset of pairwise order-incomparable elements of $S$ is finite. Unless stated otherwise, in this paper, $S$ will always be a commutative monoid, the operation of $S$ shall be denoted additively and the neutral element by 0 . The following definition is due to $[9,13,18]$.

Let $R$ be a ring, $(S, \leq)$ a strictly ordered monoid (that is, $(S, \leq)$ is an ordered monoid such that if $s, s^{\prime}, t \in S$ and $s<s^{\prime}$, then $s+t<s^{\prime}+t$ ), and $\omega: S \longrightarrow \operatorname{End}(R)$ a monoid homomorphism. For any $s \in S$, let $\omega_{s}$ denote the image of $s$ under $\omega$, that is $\omega_{s}=\omega(s)$. Consider the set $A$ of all maps $f: S \longrightarrow R$ whose support $\operatorname{supp}(f)=\{s \in S \mid f(s) \neq 0\}$ is artinian and narrow. Then for any $s \in S$ and $f, g \in A$ the set

$$
X_{s}(f, g)=\{(u, v) \in S \times S \mid u+v=s, f(u) \neq 0, g(v) \neq 0\}
$$

is finite. This fact allows to define the operation of convolution as follows:

$$
(f g)(s)=\sum_{(u, v) \in X_{s}(f, g)} f(u) \omega_{u}(g(v)), \quad \text { if } \quad X_{s}(f, g) \neq \emptyset
$$

and $(f g)(s)=0$ if $X_{s}(f, g)=\emptyset$. With this operation and pointwise addition, $A$ becomes a ring, which is called the ring of skew generalized power series with coefficients in $R$ and exponents in $S$, and we denote it by $\left[\left[R^{S, \leq}, \omega\right]\right]$.

Let $(S, \leq)$ be a strictly totally ordered monoid which is also $\operatorname{artinian,} M$ a right $R$-module and $\omega: S \longrightarrow \operatorname{Aut}(R)$ a monoid homomorphism. We let $B$ be the set of all maps $\varphi: S \longrightarrow M$ such that the $\operatorname{set} \operatorname{supp}(\varphi)=\{s \in S \mid \varphi(s) \neq 0\}$ is finite. Now $B$ can be turned into a right $\left[\left[R^{S, \leq}, \omega\right]\right]$-module. The addition in $B$ is componentwise and the scalar multiplication is defined as follows:

$$
(\varphi f)(s)=\sum_{t \in S} \varphi(s+t) \omega_{s+t}^{-1}(f(t)) \quad \text { for every } s \in S,
$$

where $f \in\left[\left[R^{S, \leq}, \omega\right]\right]$ and $\varphi \in B$. Then, by [10, Lemma 2.1], $\operatorname{supp}(\varphi f)$ is finite, and so $\varphi f$ belongs to $B$.

Suppose that $f, g \in\left[\left[R^{S, \leq}, \omega\right]\right], \varphi \in B$ and $s \in S$. Then

$$
\begin{aligned}
((\varphi f) g)(s) & =\sum_{v \in S}(\varphi f)(v+s) \omega_{v+s}^{-1}(g(v)) \\
& =\sum_{v \in S}\left(\sum_{u \in S} \varphi(u+v+s) \omega_{u+v+s}^{-1}(f(u))\right) \omega_{v+s}^{-1}(g(v))
\end{aligned}
$$




$$
=\sum_{u \in S} \sum_{v \in S} \varphi(u+v+s) \omega_{v+s}^{-1}\left(\omega_{u}^{-1}(f(u)) g(v)\right),
$$

and

$$
\begin{aligned}
(\varphi(f g))(s) & =\sum_{t \in S} \varphi(t+s) \omega_{t+s}^{-1}((f g)(t)) \\
& =\sum_{t \in S} \varphi(t+s) \omega_{t+s}^{-1}\left(\sum_{(u, v) \in X_{t}(f, g)} f(u) \omega_{u}(g(v))\right) \\
& =\sum_{(u, v) \in X} \varphi(u+v+s) \omega_{u+v+s}^{-1}\left(f(u) \omega_{u}(g(v))\right) \\
& =\sum_{(u, v) \in X} \varphi(u+v+s) \omega_{v+s}^{-1}\left(\omega_{u}^{-1}(f(u)) g(v)\right),
\end{aligned}
$$

where $X=\bigcup_{t \in S} X_{t}(f, g)$. Thus, $(\varphi f) g=\varphi(f g)$. Now, it is easy to see that $B$ becomes a right $\left[\left[R^{S, \leq}, \omega\right]\right]$-module, which we call the generalized inverse polynomial module over $\left[\left[R^{S, \leq}, \omega\right]\right]$, and denote it by $\left[M^{S, \leq}\right]$. The elements of $\left[M^{S, \leq}\right]$ are called generalized inverse polynomials with coefficients in $M$ and exponents in $S$.

For example, if $\omega_{s}=1$, the identity map of $R$ for every $s \in S$, then $\left[M^{S, \leq}\right]_{\left[\left[R^{S, \leq, \omega]}\right]\right.}=\left[M^{S, \leq}\right]_{\left[\left[R^{S, \leq}\right]\right]}$, the generalized inverse polynomial module in the sense of Liu $[6,7,8,10,11]$. In this situation, if we take $S=\mathbb{N} \cup\{0\}$, and

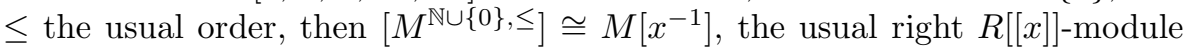
introduced in $[14,15]$, which is also called the Macaulay-Northcott module in $[16,17]$. Let $\alpha$ be a ring automorphism of $R, S=\mathbb{N} \cup\{0\}$ be endowed with the usual order and define $\omega: S \longrightarrow \operatorname{Aut}(R)$ via $\omega_{k}=\alpha^{k}$ for every $k \in \mathbb{N} \cup\{0\}$ (where $\alpha^{0}=1$, the identity map of $R$ ). Then $\left[\left[R^{S, \leq}, \omega\right]\right]=R[[x ; \alpha]]$, and $\left[M^{\mathbb{N} \cup\{0\}, \leq]}=M\left[x^{-1}\right]\right.$, the inverse polynomial modules over skew power series rings $R[[x ; \alpha]]$.

We shall henceforth assume that $(S, \leq)$ is a strictly totally ordered monoid which is also artinian. In this situation, by [10], $0 \leq s$ for any $s \in S$. This fact will be often used in our discussions. Also, in this case, for any $0 \neq f \in$ $\left[\left[R^{S,}, \omega\right]\right], \operatorname{supp}(f)$ has a minimal element, we denote it by $\pi(f)$, and for any $0 \neq \varphi \in\left[M^{S, \leq}\right], \operatorname{supp}(\varphi)$ has a maximal element, we denote it by $\sigma(\varphi)$.

In the finial of this section, we explain some notations and facts involved. To any $r \in R$ and any $s \in S$ we associate the maps $\lambda_{r}^{s} \in\left[\left[R^{S, \leq}, \omega\right]\right]$ defined by

$$
\lambda_{r}^{s}(x)= \begin{cases}r, & \text { if } x=s, \\ 0, & \text { if } x \neq s .\end{cases}
$$

In particular, denote $\lambda_{r}^{0}=c_{r}$, and $\lambda_{1}^{s}=e_{s}$. For any $m \in M$ and any $s \in S$, we define $\phi_{s, m} \in\left[M^{S, \leq}\right]$ via

$$
\phi_{s, m}(x)=\left\{\begin{array}{cc}
m, & \text { if } x=s, \\
0, & \text { if } x \neq s .
\end{array}\right.
$$


For any $s \in S$, set $G_{s}=\left\{\phi_{s, m} \mid m \in M\right\}$. Then $G_{s}$ is a right $R$-module by the right $R$-action $\phi_{s, m} r=\phi_{s, m} c_{r}$, and there exists an isomorphism of right $R$-modules $\alpha_{s}: M \longrightarrow G_{s}$ defined by $\alpha_{s}(m)=\phi_{s, m}$.

For any $\varphi \in\left[M^{S, \leq}\right]$, since $\operatorname{supp}(\varphi)$ is finite, $\varphi$ can be written as

$$
\varphi=\sum_{s \in S} \phi_{s, \varphi(s)} \text {. }
$$

This fact will also be used freely in our next discussions.

\section{Uniform dimension}

Let us first recall the notion of uniform dimension, often abbreviated by "u.dim".

Definition 2.1. We say that $M_{R}$ has uniform dimension $n$, if there is an essential submodule $V_{R} \leq M_{R}$ that is a direct sum of $n$ uniform submodules. We write u.dim $\left(M_{R}\right)=n$. If no such integer $n$ exists, we write u.dim $\left(M_{R}\right)=$ $\infty$.

An intuitive description of uniform dimension is perhaps best reflected by the following result [5, Corollary 6.6].

Lemma 2.2. For any nonzero module $M_{R}$,

$u \cdot \operatorname{dim}\left(M_{R}\right)=\sup \left\{k \mid M_{R}\right.$ contains a direct sum of $k$ nonzero submodules $\}$.

One checks easily that $\mathrm{u} \cdot \operatorname{dim}\left(M_{R}\right)=0$ if and only if $M_{R}=0$, and that u. $\operatorname{dim}\left(M_{R}\right)=1$ if and only if $M_{R}$ is uniform. Also, it is clearly possible for u. $\operatorname{dim}\left(M_{R}\right)$ to be infinite. In fact, we can characterize this situation as well [5, Proposition 6.4].

Lemma 2.3. A module $M_{R}$ has infinite uniform dimension if and only if $M_{R}$ contains an infinite direct sum of nonzero submodules.

The proof of the main result of this section relies on some elementary initial results.

Lemma 2.4. Let $(S, \leq)$ be a strictly totally ordered monoid which is also artinian, $\omega: S \longrightarrow \operatorname{Aut}(R)$ a monoid homomorphism and $N$ a submodule of $M_{R}$. Then $N$ is a uniform submodule of $M$ if and only if $\left[N^{S, \leq}\right]$ is a uniform submodule of $\left[M^{S, \leq}\right]$.

Proof. $\Longrightarrow)$ Let $0 \neq \varphi_{1}, \varphi_{2} \in\left[N^{S, \leq}\right]$, and assume that $\sigma\left(\varphi_{1}\right)=s_{1}, \sigma\left(\varphi_{2}\right)=$ $s_{2}$. Then $\varphi_{1}\left(s_{1}\right) R \cap \varphi_{2}\left(s_{2}\right) R \neq 0$ since $N$ is a uniform submodule of $M$. Since $\omega_{s_{1}}, \omega_{s_{2}} \in \operatorname{Aut}(R)$, we may select $r_{1}, r_{2} \in R$ so that $\varphi_{1}\left(s_{1}\right) \omega_{s_{1}}^{-1}\left(r_{1}\right)=$ $\varphi_{2}\left(s_{2}\right) \omega_{s_{2}}^{-1}\left(r_{2}\right) \neq 0$. Then for any $s \in S$,

$$
\left(\varphi_{i} \lambda_{r_{i}}^{s_{i}}\right)(s)=\sum_{x \in S} \varphi_{i}(x+s) \omega_{x+s}^{-1}\left(\lambda_{r_{i}}^{s_{i}}(x)\right)=\left\{\begin{array}{cl}
\varphi_{i}\left(s_{i}\right) \omega_{s_{i}}^{-1}\left(r_{i}\right), & s=0 \\
0, & s \neq 0
\end{array}\right.
$$


Thus $0 \neq \varphi_{1} \lambda_{r_{1}}^{s_{1}}=\varphi_{2} \lambda_{r_{2}}^{s_{2}} \in \varphi_{1}\left[\left[R^{S, \leq, \omega]}\right] \cap \varphi_{2}\left[\left[R^{S, \leq, \omega]}\right]\right.\right.$. Hence $\left[N^{S, \leq}\right]$ is a uniform submodule of $\left[M^{S, \leq}\right]$.

$\Longleftarrow$ Let $0 \neq n_{1}, n_{2} \in N$. Then $0 \neq \phi_{0, n_{i}} \in\left[N^{S, \leq}\right], i=1,2$. Since $\left[N^{S, \leq}\right]$ is a uniform submodule of $\left[M^{S, \leq}\right], \phi_{0, n_{1}}\left[\left[R^{S, \leq}, \omega\right]\right] \cap \phi_{0, n_{2}}\left[\left[R^{S, \leq}, \omega\right]\right] \neq 0$. Let $\phi_{0, n_{1}} f_{1}=\phi_{0, n_{2}} f_{2} \neq 0$. Then for any $s \in S$,

$$
\left(\phi_{0, n_{i}} f_{i}\right)(s)=\sum_{x \in S} \phi_{0, n_{i}}(x+s) \omega_{x+s}^{-1}\left(f_{i}(x)\right)=\left\{\begin{array}{cl}
n_{i} f_{i}(0), & s=0 \\
0, & s \neq 0 .
\end{array}\right.
$$

Thus $n_{1} f_{1}(0)=n_{2} f_{2}(0) \neq 0$. So $n_{1} R \cap n_{2} R \neq 0$. Hence $N$ is a uniform submodule of $M$.

Lemma 2.5. Let $(S, \leq)$ be a strictly totally ordered monoid which is also artinian, $\omega: S \longrightarrow \operatorname{Aut}(R)$ a monoid homomorphism and $N$ a submodule of $M_{R}$. Then $N$ is an essential submodule of $M$ if and only if $\left[N^{S, \leq}\right]$ is an essential submodule of $\left[M^{S, \leq}\right]$.

Proof. $\Longrightarrow$ Let $0 \neq \varphi \in\left[M^{S, \leq}\right]$, and assume that $\sigma(\varphi)=s$. Then there exists $r \in R$ such that $0 \neq \varphi(s) \omega_{s}^{-1}(r) \in N$ since $N$ is an essential submodule of $M$ and $\omega_{s} \in \operatorname{Aut}(R)$. Then for any $x \in S$,

$$
\left(\varphi \lambda_{r}^{s}\right)(x)=\sum_{y \in S} \varphi(x+y) \omega_{x+y}^{-1}\left(\lambda_{r}^{s}(y)\right)=\left\{\begin{array}{cl}
\varphi(s) \omega_{s}^{-1}(r) \in N, & x=0 \\
0, & x \neq 0 .
\end{array}\right.
$$

Thus $0 \neq \varphi \lambda_{r}^{s} \in\left[N^{S, \leq}\right]$. Hence $\left[N^{S, \leq}\right]$ is an essential submodule of $\left[M^{S, \leq}\right]$.

$\Longleftarrow)$ Let $0 \neq m \in M$. Then $0 \neq \phi_{0, m} \in\left[M^{S, \leq}\right]$. Since $\left[N^{S, \leq}\right]$ is an essential submodule of $\left[M^{S, \leq}\right]$, there exists an $f \in\left[\left[R^{S, \leq}, \omega\right]\right]$ such that $0 \neq \phi_{0, m} f \in$ $\left[N^{S,}\right]$. Then for any $x \in S$,

$$
\left(\phi_{0, m} f\right)(x)=\sum_{y \in S} \phi_{0, m}(x+y) \omega_{x+y}^{-1}(f(y))=\left\{\begin{array}{cl}
m f(0), & x=0 \\
0, & x \neq 0 .
\end{array}\right.
$$

Thus $0 \neq m f(0) \in N$. Hence $N$ is an essential submodule of $M$.

Now, we can prove the main result of this section.

Theorem 2.6. Let $(S, \leq)$ be a strictly totally ordered monoid which is also artinian and $\omega: S \longrightarrow \operatorname{Aut}(R)$ a monoid homomorphism. Then for any right $R$-module $M$, we have

$$
\text { u.dim }\left(\left[M^{S, \leq}\right]_{\left[\left[R^{S, \leq, \omega]}\right]\right.}\right)=\mathrm{u} \cdot \operatorname{dim}\left(M_{R}\right) .
$$

Proof. Assume that u.dim $\left(M_{R}\right)=n<\infty$. By definition, we can find uniform submodules $N_{1}, N_{2}, \ldots, N_{n}$ of $M$ such that $N_{1} \bigoplus N_{2} \bigoplus \cdots \bigoplus N_{n}$ is an essential submodule of $M$. By Lemma 2.4 and Lemma 2.5, $\left[N_{1}^{S, \leq}\right],\left[N_{2}^{S, \leq}\right], \ldots,\left[N_{n}^{S, \leq}\right]$ are uniform submodules of $\left[M^{S, \leq}\right]$, and $\left[N_{1}^{S, \leq}\right] \bigoplus\left[N_{2}^{S, \leq}\right] \bigoplus \cdots \bigoplus\left[N_{n}^{S, \leq}\right]$ is an essential submodule of $\left[M^{S, \leq}\right]$. Thus u.dim $\left(\left[M^{S, \leq}\right]_{\left[\left[R^{S, \leq, \omega]}\right]\right.}\right)=n$. 
If $\operatorname{u} \cdot \operatorname{dim}\left(M_{R}\right)=\infty$. Then, by Lemma 2.3, there exist nonzero submodules $N_{1}, N_{2}, \ldots$ of $M$ such that $\bigoplus_{i=1}^{\infty} N_{i} \leq M$. Thus $0 \neq\left[N_{i}^{S, \leq}\right] \leq\left[M^{S, \leq}\right]$, and $\bigoplus_{i=1}^{\infty}\left[N_{i}^{S, \leq}\right] \leq\left[M^{S, \leq}\right]$. This means that u.dim $\left(\left[M^{S, \leq}\right]_{\left[\left[R^{S, \leq, \omega]}\right]\right.}\right)=\infty$.

Therefore, u.dim $\left(\left[M^{S, \leq}\right]_{\left[\left[R^{S,}, \omega\right]\right]}\right)=\operatorname{u} \cdot \operatorname{dim}\left(M_{R}\right)$.

Corollary 2.7. Let $\alpha \in \operatorname{Aut}(R)$. Then for any right $R$-module $M$, we have

$$
\text { u.dim }\left(M\left[x^{-1}\right]_{R[[x ; \alpha]]}\right)=\mathrm{u} \cdot \operatorname{dim}\left(M_{R}\right) .
$$

Any submodule of the additive monoid $\mathbb{N} \cup\{0\}$ is called a numerical monoid.

Corollary 2.8. Let $\alpha \in \operatorname{Aut}(R), S$ a numerical monoid with the usual natural order of $\mathbb{N} \cup\{0\}$ and define $\omega: S \longrightarrow \operatorname{Aut}(R)$ via $\omega_{k}=\alpha^{k}$ for every $k \in S$. Then for any right $R$-module $M$, we have

$$
\text { u.dim }\left(\left[M^{S, \leq}\right]_{\left[\left[R^{S, \leq, \omega]}\right]\right.}\right)=\mathrm{u} \cdot \operatorname{dim}\left(M_{R}\right) .
$$

Let $\alpha$ and $\beta$ be ring automorphisms of $R$ such that $\alpha \beta=\beta \alpha$. Let $S=$ $(\mathbb{N} \cup\{0\}) \times(\mathbb{N} \cup\{0\})$ be endowed with the lexicographic order, or the reverse lexicographic order, or the product order of the usual order of $\mathbb{N} \cup\{0\}$, and define $\omega: S \longrightarrow \operatorname{Aut}(R)$ via $\omega(m, n)=\alpha^{m} \beta^{n}$ for any $m, n \in \mathbb{N} \cup\{0\}$. Then $\left[\left[R^{S, \leq}, \omega\right]\right]=R[[x, y ; \alpha, \beta]]$ and $\left[M^{S, \leq}\right]=M\left[x^{-1}, y^{-1}\right]$, in which

$$
\left(a x^{i} y^{j}\right)\left(b x^{p} y^{q}\right)=a \alpha^{i} \beta^{j}(b) x^{i+p} y^{j+q},
$$

where $i, j, p, q \in \mathbb{N} \cup\{0\}$ and $a, b \in R$, and

$$
\left(m x^{-i} y^{-j}\right)\left(r x^{p} y^{q}\right)=\left\{\begin{array}{cc}
m \alpha^{-i} \beta^{-j}(r) x^{-i+p} y^{-j+q}, & p \leq i, q \leq j \\
0, & \text { otherwise }
\end{array}\right.
$$

where $i, j, p, q \in \mathbb{N} \cup\{0\}$ and $r \in R, m \in M$.

Corollary 2.9. For any right $R$-module $M$, we have

$$
\text { u.dim }\left(M\left[x^{-1}, y^{-1}\right]_{R[[x, y ; \alpha, \beta]]}\right)=\mathrm{u} \cdot \operatorname{dim}\left(M_{R}\right) \text {. }
$$

Corollary 2.10. Let $(S, \leq)$ be a strictly totally ordered monoid which is also artinian. Then for any right $R$-module $M$, we have

$$
\text { u.dim }\left(\left[M^{S, \leq}\right]_{\left[\left[R^{S,} \leq\right]\right]}\right)=\mathrm{u} \cdot \operatorname{dim}\left(M_{R}\right) .
$$

If $S$ is the multiplicative monoid $(\mathbb{N}, \cdot)$, endowed with the usual order $\leq$, then $\left[\left[R^{(\mathbb{N}, \cdot), \leq}\right]\right]$ is the ring of arithmetical functions with values in $R$, endowed with the Dirichlet convolution:

$$
(f g)(n)=\sum_{d \mid n} f(d) g(n / d) \quad \text { for each } n \geq 1 .
$$

If $M$ is a right $R$-module, then the right $\left[\left[R^{(\mathbb{N}, \cdot), \leq}\right]\right]-$ module $\left[M^{(\mathbb{N}, \cdot), \leq}\right]$ is the set $\left\{\sum_{i=1}^{n} m_{i} x^{-i} \mid m_{i} \in M, i=1,2, \ldots, n, n \in \mathbb{N}\right\}$ with scalar multiplication as below:

$$
\left(\sum_{j \geq 1} m_{j} x^{-j}\right)\left(\sum_{i \geq 1} r_{i} x^{i}\right)=\sum_{j \geq 1}\left(\sum_{i \geq 1} m_{i \cdot j} r_{i}\right) x^{-j}
$$


where $\sum_{i \geq 1} r_{i} x^{i} \in\left[\left[R^{(\mathbb{N}, \cdot), \leq}\right]\right]$ and $\sum_{j \geq 1} m_{j} x^{-j} \in\left[M^{(\mathbb{N}, \cdot), \leq}\right]$.

Corollary 2.11. For any right $R$-module $M$, we have

$$
\operatorname{u.dim}\left(\left[M^{(\mathbb{N}, \cdot), \leq}\right]_{\left[\left[R^{(\mathbb{N}, \cdot), \leq}\right]\right]}\right)=\operatorname{u} \cdot \operatorname{dim}\left(M_{R}\right) .
$$

\section{Couniform dimension}

As a dual of the uniform dimension of a module, Varadarajan introduced the couniform dimension of a module in his two 1979 papers [21] and [19], and obtained a number of results on couniform dimension. In [1, 2], Annin obtained result on the couniform dimension of the inverse polynomial module $M\left[x^{-1}\right]$ over skew polynomial rings. In this section, we study the couniform dimension of generalized inverse polynomial modules over skew generalized power series rings. At this point, we have everything we will need for our purposes firstly.

Definition 3.1. For any module $M_{R}$, we define $\operatorname{corank}\left(M_{R}\right)=\sup \left\{k \mid M_{R}\right.$ surjects onto a direct sum of $k$ nonzero modules $\}$. In particular, $\operatorname{corank}(0)=0$.

A nonzero module $M_{R}$ is called hollow if the sum of any two of its proper submodules is also a proper submodule. It is easy to see that $\operatorname{corank}\left(M_{R}\right)=1$ if and only if $M_{R}$ is hollow. We next record a few of the basic results from [21] and [19] (or see [1, 2]) on couniform dimension that will be needed below. We recall that a submodule $K$ of $M_{R}$ is called superfluous (or small) if, for every submodule $N \leq M_{R}$ with $N+K=M$, we have $N=M$. We will indicate that $K$ is a superfluous submodule of $M$ by the notation $K \ll M$.

Lemma 3.2. (1) For any right $R$-module $M, \operatorname{corank}\left(M_{R}\right)=k<\infty$ if and only if there exists a surjection $\varphi: M \longrightarrow \bigoplus_{i=1}^{k} N_{i}$ with $\operatorname{Ker}(\varphi) \ll M$ and all $N_{i}$ hollow.

(2) Let $M$ be a right $R$-module and $N \leq M$. Then corank $(M / N)_{R} \leq$ $\operatorname{corank}\left(M_{R}\right)$. In particular, if $N \ll M$, then $\operatorname{corank}\left(M_{R}\right)=\operatorname{corank}(M / N)_{R}$.

(3) Given right $R$-modules $M_{1}, M_{2}, \ldots, M_{n}$, we have $\operatorname{corank}\left(\bigoplus_{i=1}^{n} M_{i}\right)=$ $\sum_{i=1}^{n} \operatorname{corank}\left(M_{i}\right)$.

For the proof of the main result of this section, there are some central lemmas that we must first establish.

Lemma 3.3. Let $(S, \leq)$ be a strictly totally ordered monoid which is also artinian, $\omega: S \longrightarrow \operatorname{Aut}(R)$ a monoid homomorphism, $M$ a right $R$-module and $P$ a maximal $R$-submodule of $\left[M^{S, \leq}\right]$. For any $s \in S$, set $P_{s}=\left\{m \in M \mid \phi_{s, m} \in\right.$ $P\}$. Then for each $s \in S$, either $P_{s}=M$ or $P_{s}$ is a maximal $R$-submodule of $M_{R}$. Moreover, there exists $s \in S$ for which the latter holds.

Proof. Clearly, $\phi_{s, m_{1}+m_{2}}=\phi_{s, m_{1}}+\phi_{s, m_{2}}$ and $\phi_{s, m} \omega_{s}(r)=\phi_{s, m r}$ for any $r \in R$ and any $m, m_{1}, m_{2} \in M$. Then it is easy to see that $P_{s}$ is an $R$-submodule of $M_{R}$. Suppose that for some $s \in S, P_{s} \neq M$. To show that $P_{s}$ is a maximal 
submodule of $M_{R}$, assume that $P_{s} \lesseqgtr N \leq M_{R}$. Let $n \in N-P_{s}$. Then $\phi_{s, n} \notin P$. Thus $\left[M^{S, \leq}\right]_{R}=P+\phi_{s, n} R$ since $P$ is a maximal $R$-submodule of $\left[M^{S, \leq}\right]$. Then, for any $m \in M$, there exist $p \in P$ and $r \in R$ such that $\phi_{s, m}=p+\phi_{s, n} r$. Thus $p=\phi_{s, m}-\phi_{s, n} r \in P$. Note that $\phi_{s, m}-\phi_{s, n} r=\phi_{s, m-n \omega_{s}^{-1}(r)}$. Thus $m-n \omega_{s}^{-1}(r) \in P_{s} \lesseqgtr N$. Hence $m \in N$, and so $N=M$. This means that $P_{s}$ is a maximal $R$-submodule of $M_{R}$. For the last assertion, assume to the contrary, meanly $P_{s}=M$ for all $s \in S$. Then for any $\varphi \in\left[M^{S, \leq}\right]$ and any $x \in S, \varphi(x) \in M=P_{x}$, and so $\phi_{x, \varphi(x)} \in P$. Hence $\varphi=\sum_{x \in S} \phi_{x, \varphi(x)} \in P$. Thus $P=\left[M^{S, \leq}\right]$, which contradicts to the hypothesis that $P$ is a maximal $R$-submodule of $\left[M^{S, \leq}\right]$.

Lemma 3.4. Let $(S, \leq)$ be a strictly totally ordered monoid which is also artinian, $\omega: S \longrightarrow \operatorname{Aut}(R)$ a monoid homomorphism, $M$ a right $R$-module and $N$ a submodule of $M$. If $R$ is a right perfect ring, then $N \ll M$ if and only if $\left[N^{S, \leq}\right] \ll\left[M^{S, \leq}\right]$.

Proof. $\Longleftarrow)$ If $N$ is not superfluous in $M$, then we can find $L \lesseqgtr M$ such that $N+L=M$. Then $\left[M^{S, \leq}\right]=\left[N^{S, \leq}\right]+\left[L^{S, \leq}\right]$, and $\left[L^{S, \leq}\right] \lesseqgtr\left[M^{S, \leq}\right]$. This contradicts to the hypothesis that $\left[N^{S, \leq}\right] \ll\left[M^{S, \leq}\right]$.

$\Longrightarrow)$ Suppose that there exists $Q \lessgtr\left[M^{S, \leq}\right]_{\left[\left[R^{S, \leq, \omega]}\right]\right.}$ with $Q+\left[N^{S, \leq}\right]=$ $\left[M^{S, \leq}\right]$. Then $Q_{R}+\left[N^{S, \leq}\right]_{R}=\left[M^{S, \leq}\right]_{R}$. Since $R$ is a right perfect ring, $Q_{R}$ is contained in a maximal submodule $P_{R} \lesseqgtr\left[M^{S, \leq}\right]_{R}$. So, $P_{R}+\left[N^{S, \leq}\right]_{R}=$ $\left[M^{S, \leq}\right]_{R}$, and it is clear that $\left[N^{S, \leq}\right]_{R} \nsubseteq P$. Thus there exists a $\varphi \in\left[N^{S, \leq}\right] \backslash P$. Note that $\varphi=\sum_{s \in S} \phi_{s, \varphi(s)}$, there are some $s \in S$ such that $\phi_{s, \varphi(s)} \notin P$. Let $P_{s}=\left\{m \in M \mid \phi_{s, m} \in P\right\}$. Then by Lemma 3.3, either $P_{s}=M$ or $P_{s}$ is a maximal $R$-submodule of $M_{R}$. Since $\varphi(s) \in N \backslash P_{s}$, so the latter option holds. Thus, since $N \nsubseteq P_{s}$, we have $M_{R}=P_{s}+N$. The fact that $P_{s} \neq M$ now implies that $N_{R}$ is not superfluous in $M_{R}$, completing the proof.

Following [3], a monoid $S$ is said to be chain if the ideals of $S$ are totally ordered by set inclusion, i.e., for any $s, t \in S$, either $s+S \subseteq t+S$ or $t+S \subseteq s+S$.

Lemma 3.5. Let $(S, \leq)$ be a strictly totally ordered monoid which is also artinian, $\omega: S \longrightarrow \operatorname{Aut}(R)$ a monoid homomorphism and $M$ a right $R$-module. If $R$ is a right perfect ring and $S$ a chain monoid, then $M$ is hollow if and only if $\left[M^{S, \leq}\right]$ is hollow.

Proof. $\Longleftarrow)$ If $M_{R}$ is not hollow, we can find $K, L \lesseqgtr M$ with $M=K+L$. Then $\left[M^{S, \leq}\right]=\left[K^{S, \leq}\right]+\left[L^{S, \leq}\right]$ with $\left[K^{S, \leq}\right],\left[L^{S, \leq}\right] \subsetneq\left[M^{S, \leq}\right]$. This contradicts to the fact that $\left[M^{S, \leq}\right]$ is hollow.

$\Longrightarrow)$ We complete by two steps.

Step 1: We show that if $M$ is simple, then $\left[M^{S, \leq}\right]$ is hollow.

For this, firstly, we show that for any $0 \neq Q \subsetneq\left[M^{S, \leq}\right]_{\left[\left[R^{S,}, \omega\right]\right]}$, there exists an $s \in S$ such that $\sigma(\varphi) \leq s$ for any $0 \neq \varphi \in Q$.

Assume the result is false, and let $0 \neq Q \lesseqgtr\left[M^{S, \leq}\right]_{\left[\left[R^{S, \leq, \omega]}\right]\right.}$ be such that for any $s \in S$, there exists $0 \neq \varphi \in Q$ with $\sigma(\varphi)>s$. Let $s \in S$, set 
$G_{s}=\left\{\phi_{s, m} \mid m \in M\right\}$. Then, $G_{s}$ is a right $R$-module and $G_{s} \cong M_{R}$. Thus, $G_{s}$ is a simple right $R$-module for any $s \in S$. We show that $G_{s} \subseteq Q$ by induction on $s \in S$.

For $s=0$, by the hypothesis, choose a $0 \neq \varphi \in Q$ such that $\sigma(\varphi)>0$. Assume that $\sigma(\varphi)=u$. Then $\varphi e_{u}=\phi_{0, \varphi(u)}$. Thus $0 \neq \phi_{0, \varphi(u)} \in Q \cap G_{0} \subseteq G_{0}$. Since $G_{0}$ is simple, $G_{0}=G_{0} \cap Q$. Hence, $G_{0} \subseteq Q$.

Now, let $0<w \in S$. Assume that for any $s<w, G_{s} \subseteq Q$. We will show that $G_{w} \subseteq Q$. By the hypothesis, there exists a $0 \neq \varphi \in Q$ such that $\sigma(\varphi)>w$. Assume that $\sigma(\varphi)=u$. Since $S$ is a chain monoid, there exists a $v \in S$ such that $u=w+v$. For any $x>w$, since $(S, \leq)$ is a strictly ordered monoid, $u=w+v<x+v$. Thus

$$
\left(\varphi e_{v}\right)(x)=\sum_{y \in S} \varphi(x+y) \omega_{x+y}^{-1}\left(e_{v}(y)\right)=\varphi(x+v)=0 .
$$

Hence $\sigma\left(\varphi e_{v}\right) \leq w$. Note that

$$
\left(\varphi e_{v}\right)(w)=\sum_{y \in S} \varphi(w+y) \omega_{\omega+y}^{-1}\left(e_{v}(y)\right)=\varphi(w+v)=\varphi(u) \neq 0 .
$$

Thus $\sigma\left(\varphi e_{v}\right)=w$. Hence

$$
\varphi e_{v}=\sum_{x \in S} \phi_{x,\left(\varphi e_{v}\right)(x)}=\phi_{w, \varphi(u)}+\sum_{x<w} \phi_{x,\left(\varphi e_{v}\right)(x)} .
$$

By the hypothesis, $\sum_{x<w} \phi_{x,\left(\varphi e_{v}\right)(x)} \in Q$. Hence

$$
0 \neq \phi_{w, \varphi(u)}=\varphi e_{v}-\sum_{x<w} \phi_{x,\left(\varphi e_{v}\right)(x)} \in Q \cap G_{w} \subseteq G_{w} .
$$

Since $G_{w}$ is a simple right $R$-module, $Q \cap G_{w}=G_{w}$, so $G_{w} \subseteq Q$.

Therefore, by transfinite induction, we have shown that for any $s \in S, G_{s} \subseteq$ $Q$. Thus, for any $\varphi \in\left[M^{S, \leq}\right]$, since $\varphi=\sum_{x \in S} \phi_{x, \varphi(x)}$, we have $\varphi \in Q$. Hence $Q=\left[M^{S, \leq}\right]$, which contradicts to the fact that $Q$ is a proper submodule of $\left[M^{S, \leq}\right]$. Therefore, for any $0 \neq Q \lesseqgtr\left[M^{S, \leq}\right]$, there exists an $s \in S$ such that $\sigma(\varphi) \leq s$ for any $0 \neq \varphi \in Q$.

Now, we show that $\left[M^{S, \leq}\right]$ is a hollow module. Let $0 \neq P, Q \lesseqgtr\left[M^{S, \leq}\right]$. Then there exists $0 \neq u \in S$ such that $\sigma(\varphi) \leq u, \sigma(\psi) \leq u$ for any $0 \neq \varphi \in P$ and any $0 \neq \psi \in Q$. Thus, for any $0 \neq m \in M$, we have $\phi_{2 u, m} \in\left[M^{S, \leq}\right] \backslash(P+$ $Q)$. This implies that $\left[M^{S, \leq}\right]$ is a hollow right $\left[\left[R^{S, \leq}, \omega\right]\right]$-module.

Step 2: We show that if $M$ is a hollow right $R$-module, then $\left[M^{S, \leq}\right]$ is a hollow right $\left[\left[R^{S, \leq}, \omega\right]\right]$-module.

Assume that there exist $Q, Q^{\prime} \lesseqgtr\left[M^{S, \leq}\right]_{\left[\left[R^{S,}, \omega\right]\right]}$ such that $Q+Q^{\prime}=\left[M^{S, \leq}\right]$. Since $R$ is a right perfect ring, $M_{R}$ has a maximal submodule, say $L$. Then $L \ll M$ since $M_{R}$ is hollow. By Lemma $3.4,\left[L^{S, \leq}\right] \ll\left[M^{S, \leq}\right]$. Hence, $\left[L^{S, \leq}\right]+Q$ and $\left[L^{S, \leq}\right]+Q^{\prime}$ are both proper submodules of $\left[M^{S, \leq}\right]$. If $\left[L^{S, \leq}\right]+Q=\left[L^{S, \leq}\right]$, then $\left[L^{S, \leq}\right]+Q^{\prime}=\left[L^{S, \leq}\right]+Q+Q^{\prime}=\left[M^{S, \leq}\right]$, a contradiction. Thus $\left[L^{S, \leq}\right] \lessgtr$ 
$\left[L^{S, \leq}\right]+Q$. Similarly, $\left[L^{S, \leq}\right] \lesseqgtr\left[L^{S, \leq}\right]+Q^{\prime}$. Hence, $\left(\left[L^{S, \leq}\right]+Q\right) /\left[L^{S, \leq}\right]$, $\left(\left[L^{S, \leq}\right]+Q^{\prime}\right) /\left[L^{S, \leq}\right]$ are all proper submodules of $\left[M^{S, \leq}\right] /\left[L^{S, \leq}\right]$, and

$$
\left(\left[L^{S, \leq}\right]+Q\right) /\left[L^{S, \leq}\right]+\left(\left[L^{S, \leq}\right]+Q^{\prime}\right) /\left[L^{S, \leq}\right]=\left[M^{S, \leq}\right] /\left[L^{S, \leq}\right] .
$$

On the other hand, since $\left[M^{S, \leq}\right] /\left[L^{S, \leq}\right] \cong\left[(M / L)^{S, \leq}\right]$, and $M / L$ is a simple right $R$-module, by Step $1,\left[(M / L)^{S, \leq}\right]$ is hollow. Hence $\left[M^{S, \leq}\right] /\left[L^{S, \leq}\right]$ is hollow, a contradiction. Now the result follows.

Now, we prove our second main result.

Theorem 3.6. Let $(S, \leq)$ be a strictly totally ordered monoid which is also artinian and $\omega: S \longrightarrow \operatorname{Aut}(R)$ a monoid homomorphism. If $R$ is a right perfect ring and $S$ is a chain monoid, then for any right $R$-module $M$, we have

$$
\operatorname{corank}\left(\left[M^{S, \leq}\right]_{\left[\left[R^{S,}, \omega\right]\right]}\right)=\operatorname{corank}\left(M_{R}\right) .
$$

Proof. Suppose first that $\operatorname{corank}\left(M_{R}\right)=n<\infty$. By Lemma 3.2(1), we have hollow $R$-modules $H_{1}, H_{2}, \ldots, H_{n}$, and a surjection $\alpha: M \longrightarrow \bigoplus_{i=1}^{n} H_{i}$ such that $\operatorname{Ker} \alpha \ll M_{R}$. Define $\beta:\left[M^{S, \leq}\right] \longrightarrow\left[\left(\bigoplus_{i=1}^{n} H_{i}\right)^{S, \leq}\right]$ via:

$$
\beta(\varphi)(s)=\alpha(\varphi(s)), \quad \forall \varphi \in\left[M^{S, \leq}\right], \forall s \in S .
$$

Since $\operatorname{supp}(\varphi)$ is finite, $\operatorname{supp}(\beta(\varphi))$ finite. Thus $\beta$ is well-defined. For any $f \in\left[\left[R^{S, \leq}, \omega\right]\right]$, any $\varphi \in\left[M^{S, \leq}\right]$ and any $s \in S$,

$$
\begin{aligned}
\beta(\varphi f)(s) & =\alpha((\varphi f)(s))=\alpha\left(\sum_{x \in S} \varphi(x+s) \omega_{x+s}^{-1}(f(x))\right) \\
& =\sum_{x \in S} \alpha\left(\varphi(x+s) \omega_{x+s}^{-1}(f(x))\right)=\sum_{x \in S} \alpha(\varphi(x+s)) \omega_{x+s}^{-1}(f(x)) \\
& =\sum_{x \in S} \beta(\varphi)(x+s) \omega_{x+s}^{-1}(f(x))=(\beta(\varphi) f)(s) .
\end{aligned}
$$

Thus $\beta(\varphi f)=\beta(\varphi) f$. Now, it is easy to see that $\beta$ is a right $\left[\left[R^{S, \leq}, \omega\right]\right]-$ homomorphism.

Let $\psi \in\left[\left(\bigoplus_{i=1}^{n} H_{i}\right)^{S, \leq}\right]$. Then for any $s \in \operatorname{supp}(\psi)$, there exists an $m_{s} \in M$ such that $\alpha\left(m_{s}\right)=\psi(s)$. Define $\varphi \in\left[M^{S, \leq}\right]$ via:

$$
\varphi(s)=\left\{\begin{array}{cc}
m_{s}, & s \in \operatorname{supp}(\psi), \\
0, & s \notin \operatorname{supp}(\psi) .
\end{array}\right.
$$

Then for any $s \in S$,

$$
\beta(\varphi)(s)=\alpha(\varphi(s))=\alpha\left(m_{s}\right)=\psi(s) .
$$


Thus $\beta(\varphi)=\psi$. Hence $\beta$ is an epimorphism. Since

$$
\begin{aligned}
\operatorname{Ker} \beta & =\left\{\varphi \in\left[M^{S, \leq}\right] \mid \beta(\varphi)=0\right\} \\
& =\left\{\varphi \in\left[M^{S, \leq}\right] \mid \alpha(\varphi(s))=0, \forall s \in S\right\} \\
& =\left\{\varphi \in\left[M^{S, \leq}\right] \mid \varphi(s) \in \operatorname{Ker} \alpha, \forall s \in S\right\} \\
& =\left[(\operatorname{Ker} \alpha)^{S, \leq}\right],
\end{aligned}
$$

so Lemma 3.4 implies that $\operatorname{Ker} \beta=\left[(\operatorname{Ker} \alpha)^{S, \leq}\right] \ll\left[M^{S, \leq}\right]$. Thus, by Lemma $3.2(2)$, we have

$$
\operatorname{corank}\left(\left[M^{S, \leq}\right]\right)=\operatorname{corank}\left(\left[M^{S, \leq}\right] / \operatorname{Ker} \beta\right)=\operatorname{corank}\left(\left[\left(\bigoplus_{i=1}^{n} H_{i}\right)^{S, \leq}\right]\right) .
$$

Note that $\left[\left(\bigoplus_{i=1}^{n} H_{i}\right)^{S, \leq}\right] \cong \bigoplus_{i=1}^{n}\left[H_{i}^{S, \leq}\right]$, we have

$$
\operatorname{corank}\left(\left[M^{S, \leq}\right]\right)=\operatorname{corank}\left(\bigoplus_{i=1}^{n}\left[H_{i}^{S, \leq}\right]\right) \text {. }
$$

Now, Lemma 3.2(3) implies that,

$$
\operatorname{corank}\left(\left[M^{S, \leq}\right]\right)=\operatorname{corank}\left(\bigoplus_{i=1}^{n}\left[H_{i}^{S, \leq}\right]\right)=\sum_{i=1}^{n} \operatorname{corank}\left(\left[H_{i}^{S, \leq}\right]\right) .
$$

Since $H_{1}, H_{2}, \ldots, H_{n}$ are all hollow modules, by Lemma $3.5,\left[H_{1}^{S, \leq}\right],\left[H_{2}^{S, \leq}\right], \ldots$, $\left[H_{n}^{S, \leq}\right]$ are hollow modules. Thus, $\operatorname{corank}\left(\left[H_{i}^{S,}\right]\right)=1, i=1,2, \ldots, n$. Hence

$$
\operatorname{corank}\left(\left[M^{S, \leq}\right]\right)=\sum_{i=1}^{n} \operatorname{corank}\left(\left[H_{i}^{S, \leq}\right]\right)=n .
$$

Secondly, if $\operatorname{corank}\left(M_{R}\right)=\infty$, then for arbitrarily large $k$, we have a surjection $\alpha_{k}: M \longrightarrow \bigoplus_{i=1}^{k} N_{i}$ with $N_{i} \neq 0$. This induces a surjection $\beta_{k}$ : $\left[M^{S, \leq}\right] \longrightarrow \bigoplus_{i=1}^{k}\left[N_{i}^{S, \leq}\right]$ for each such $k$, which shows that $\operatorname{corank}\left(\left[M^{S, \leq}\right]\right)=$ $\infty$.

Therefore, $\operatorname{corank}\left(\left[M^{S, \leq}\right]_{\left[\left[R^{S, \leq}\right]\right]}\right)=\operatorname{corank}\left(M_{R}\right)$.

Remark ([2, Example 2.7]). implied that the corank of $M\left[x^{-1}\right]$ may not equivalent to the corank of $M_{R}$ without the assumption that $R$ is a right perfect ring. Thus, the right perfect condition of the ring $R$ is also essential in Theorem 3.6.

Corollary 3.7. Let $R$ be a right perfect ring and $\alpha \in \operatorname{Aut}(R)$. Then for any right $R$-module $M$, we have

$$
\operatorname{corank}\left(M\left[x^{-1}\right]_{R[[x ; \alpha]]}\right)=\operatorname{corank}\left(M_{R}\right) .
$$

Corollary 3.8. Let $R$ be a right perfect ring, $\alpha \in \operatorname{Aut}(R)$ and $S$ a numerical monoid with the usual natural order of $\mathbb{N} \cup\{0\}$ and define $\omega: S \longrightarrow \operatorname{Aut}(R)$ via $\omega_{k}=\alpha^{k}$ for every $k \in S$. Then for any right $R$-module $M$, we have

$$
\operatorname{corank}\left(\left[M^{S, \leq}\right]_{\left[\left[R^{S,} \leq, \omega\right]\right]}\right)=\operatorname{corank}\left(M_{R}\right) \text {. }
$$


Corollary 3.9. If $R$ is a right perfect ring, then for any right $R$-module $M$, we have

$$
\operatorname{corank}\left(M\left[x^{-1}, y^{-1}\right]_{R[[x, y ; \alpha, \beta]]}\right)=\operatorname{corank}\left(M_{R}\right) \text {. }
$$

Acknowledgments. The author wishes to thank the referee for his/her valuable suggestions. This research is supported by the National Natural Science Foundation of China (10961021) and NWNU-LKQN-11-6.

\section{References}

[1] S. Annin, Associated and attached primes over noncommutative rings, Ph. D. Diss., University of California at Berkeley, 2002.

[2] _ Couniform dimension over skew polynomial rings, Comm. Algebra 33 (2005), no. 4, 1195-1204.

[3] M. Ferrero, R. Mazurek, and A. Sant'Ana, On right chain semigroups, J. Algebra 292 (2005), no. 2, 574-584.

[4] P. Grzeszczuk, Goldie dimension of differential operator rings, Comm. Algebra 16 (1988), no. 4, 689-701.

[5] T. Y. Lam, Lectures on Modules and Rings, Graduate Texts in Mathematics volume 189, Springer-Verlag, Berlin-Heidelberg-New York, 1999.

[6] Z. K. Liu, Endomorphism rings of modules of generalized inverse polynomials, Comm. Algebra 28 (2000), no. 2, 803-814.

[7] - Injectivity of modules of generalized inverse polynomials, Comm. Algebra 29 (2001), no. 2, 583-592.

[8] Injective precover and modules of generalized inverse polynomials, Chin. Ann. Math. Ser. B 25 (2004), no. 1, 129-138.

[9] _ Triangular matrix representations of rings of generalized power series, Acta Math. Sin. (Engl. Ser.) 22 (2006), no. 4, 989-998.

[10] Z. K. Liu and H. Cheng, Quasi-duality for the rings of generalized power series, Comm. Algebra 28 (2000), no. 3, 1175-1188.

[11] Z. K. Liu and Y. Fan, Co-Hopfian modules of generalized inverse polynomials, Acta Math. Sin. (Engl. Ser.) 17 (2001), no. 3, 431-436.

[12] J. Matczuk, Goldie rank of Ore extensions, Comm. Algebra 23 (1995), no. 4, 1455-1471.

[13] R. Mazurek and M. Ziembowski, Uniserial rings of skew generalized power series, J. Algebra 318 (2007), no. 2, 737-764.

[14] A. S. McKerrow, On the injective dimension of modules of power series, Quart. J. Math. Oxford Ser. (2) 25 (1974), 359-368.

[15] D. G. Northcott, Injective envelopes and inverse polynomials, London Math. Soc. 8 (1974), 290-296.

[16] S. Park, The Macaulay-Northcott functor, Arch. Math. 63 (1994), no. 3, 225-230.

[17] _ Inverse polynomials and injective covers, Comm. Algebra 21 (1993), no. 12, $4599-4613$.

[18] P. Ribenboim, Semisimple rings and von Neumann regular rings of generalized power series, J. Algebra 198 (1997), no. 2, 327-338.

[19] B. Sarath and K. Varadarajan, Dual Goldie dimension II, Comm. Algebra 7 (1979), no. 17, 1885-1899.

[20] R. C. Shock, Polynomial rings over finite dimensional rings, Pacific J. Math. 42 (1972), 251-257.

[21] K. Varadarajan, Dual Goldie dimension, Comm. Algebra 7 (1979), no. 6, 565-610.

[22] _ On a theorem of Shock, Comm. Algebra 10 (1982), no. 20, 2205-2222.

[23] - Dual Goldie dimension of certain extension rings, Comm. Algebra 10 (1982), no. $20,2223-2231$. 
[24] R. Y. Zhao and Z. K. Liu, Artinness of generalized Macaulay-Northcott modules, Comm. Algebra 37 (2009), no. 2, 525-531.

College of Economics and Management

NorThWEST Normal UNIVERSiTy

LANZHou 730070, P. R. China

E-mail address: renyuzhao026@gmail.com 